\title{
The design and application of battlefield dynamics based on large data
}

\author{
Lu Guilin ${ }^{1, a, *}$, Wang Shaohong, \\ ${ }^{1}$ GuangXi University of science and Technology, No. 268 Donghuan Road, LiuZhou,P. R. China,545006 \\ ${ }^{2}$ Air Force No.95275, Liuzhou, 545006, P. R. China,545006 \\ a kejichenguodahuei@126.com, ${ }^{b}$ juashangyuhua@163.com
}

Keywords: Cloud services; The ESB service bus; SmartGWT technology; ITSM

\begin{abstract}
For multi-target tracking and warning, cloud computing provides virtualization physical resource of cloud services, according to a single target exists in the process of drawing the boundary of the vertex and inconsistent problem, the service bus ESB using SmartGWT technology, automatic find infrastructure cloud services network topology, combined with the ITSM for key process, the simulation results in the form of graphics rendering to the three-dimensional space, service of battlefield visualization, viewing and management.
\end{abstract}

\section{Introduction}

Service-oriented architecture SOA, the local service physical land, sea and air transportation network are based on the energy grid ++ and developed a platform of entity, a remote service using modular application program interface of Web services

ITSM manages the big data storage of multiple sensors, implements data compression, collects and monitors, and alerts when necessary, and completes the process of service object and configuration management

For this service access for distributed enterprise service bus (ESB, the switches in the network layer topology discovery has jurisdiction subnet topology relationship, and display a variety of network topology is introduced into service management in a timely manner.

For this service access for distributed enterprise service bus (ESB, the switches in the network layer topology discovery,we has jurisdiction subnet topology relationship, and display a variety of network topology is introduced into service management in a timely manner.

The interface of different service object the time-varying characteristics of the electric equipment, the system provides each user's use of the wireless resources, and from the Angle of time domain we analysis to extend the time of the OFDM symbol, the big data signal to parallel data flow.

From the point of view of frequency domain, is use frequency selective channel into a set of parallel channel interface,it provide priority to submit an application user interface allocation dynamic resource allocation methods, and meet the needs of the land, sea and air electromagnetic scattering of battlefield is generated.

\section{Manuscript Preparation}

The offshore target airborne antenna of one of the infrastructure services establishes the mathematical model at random process, the ACU controller is connected with the PC's hardware, and the PC serial port receives the signal. Although no ship can be priori in any time, in the tracking process, the values of the sea position are subject to the statistical law of the correlation between square wave and sine wave ${ }^{[1]}$

\subsection{System virtualization access to cloud service platform}

Measure the change of the ship gesture, antenna controller according to the characteristics of planar antenna array direction with the deviation Angle error of ship motion relative to the satellite, array element position vector can be regarded as digital matrix of spatial filter group. 


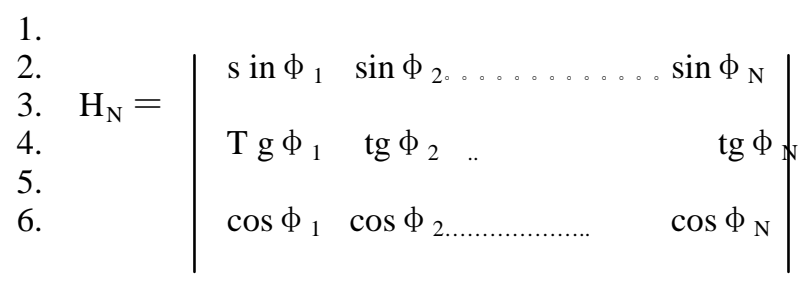

The Angle of array distribution of antenna array: $\frac{n \pi}{2 N}$

In where, $n=1,2,3, \ldots . . \mathrm{N}$

According to the error adjusting azimuth Angle and elevation Angle of the antenna is stationary random signals, leading to heading vector for antenna controller

$$
\sum_{k=11}^{N} \sum_{l=1}^{N} m_{k}^{*} T_{x x}(k-l+1) m_{l}
$$

Correlation function sequence $T_{x x}(k-l+1)$ It is semi-positive and the weighted coefficient varies with the horizontal and pitch adjustment, and the output meets the Langevin equation, which finally enables the antenna to automatically detect and control automatically.

The radar detection must make the RCS improve to the maximum. The main function of the RCS tribute on the NUBBS is the standing point and the stationary phase ${ }^{[2]}$

$\nabla .(m \nabla u)+m u=c d u$

Assume that the bandwidth of RAM required for $\left(f_{\min }, f_{\max }\right)$, The boundary condition satisfies the Dirichlet condition, and the objective function of the bionic optimization algorithm is

$$
\begin{aligned}
& \Delta r+f(r)=k r^{2} \\
& r=e^{-i k x}, \frac{\partial e}{\partial n}=i k x
\end{aligned}
$$

The detection target land tank, artillery and other regional distribution

Flying target track is the determination of the same data variables, a unified method of moments, then matrix compression, due to the operation on the matrix adaptively extract the row and column elements, which has more prominent compression efficiency. It is concluded that the attitude of the inertial navigation, flight distance and speed error state parameter equation, and the geographic coordinates and the relative coordinate system between the rotating angular velocity and the coordinate transformation matrix. Toward a greater likelihood probability matrix filling, at the same time, based on the pseudo-range and pseudo-range rate of integrated navigation model estimates the transition probability matrix of solving matrix equation, and then realize the observation matrix vector motor $4 \mathrm{~d}$ ship motion prediction model. Will MSIMMPDA optimization algorithm based on markov theory, and USES the theory of moment invariants of aircraft target feature extraction, combined with sequential fusion method, used for real-time fusion of target tracking.

Naval battle field electromagnetic situation generated framework for underwater target by horizontal electromagnetic interference, and detect the GPS signal is not accurate, under the condition of information is sparse, in terms of posture alignment, the rotating modulation technology with flying target coordinate system and the Angle between the navigation coordinates the same carrier attitude matrix for error correction, achieve the position calibration without back.

Using multiple unbiased estimations, the optimization of kalman filtering is carried out between the integral of motion acceleration and acceleration of gravity

By position and velocity calibration, in view of the underwater target diving time is long, the electromagnetic field special characteristic, and MEMS inertial sensors based on DSP is studied, and the simulated data and digital processing of these information, so as to determine the carrier of the diving depth, average speed and Angle of rotation, such as navigation information, navigation 
way has the ability to resist electromagnetic interference attitude sensing method, attitude measurement accuracy up to 3 degrees

Due to underwater target is divided into the main body and drag the part, the presence of discontinuous signal, doppler log beidou satellite system as the auxiliary sensors, actual navigation system by the combination of inertial sensors and has the function of correcting navigation devices, all sensor information fusion optimization algorithm, so as to achieve the goal of complementary information

The output data of the output attitude of the combined navigation device is the data perturbation of the inertial measurement unit and the output data of the carrier coordinate system, and the acceleration signal after the MEMS method is processed ${ }^{[3]}$

$$
a=\frac{\rho}{N_{i}} \Psi x+z
$$

In the formula, $\rho$ is the average SNR, $\mathrm{z}$ is the noise in the water, and the rotation direction is the same direction matrix: the execution of strictly diagonally dominant treatment is after

$$
c_{m}^{n}=\left|\begin{array}{ccl}
x \cos \theta+\mathrm{z} & 0 & \sin \theta+\mathrm{z} \\
0 & 1 & 0 \\
-\sin \theta+\mathrm{z} & 0 & x \cos \theta+\mathrm{z}
\end{array}\right|
$$

The rotary modulation technique is the final comprehensive evaluation:

$$
C=c_{m-1}^{n-1} C_{m-2}^{n-2} \ldots . . c_{2}^{2} c_{m}^{n}
$$

\subsection{Cloud service resource management and monitoring design process}

Cloud computing big data model is the first layer include electromagnetic scattering software and interface, etc, the second layer is based on $4 \mathrm{~g}, 5 \mathrm{~g}$ communications platform services, cloud services, the third layer is the hardware on the fourth floor is virtualization services, general simulation platform based on HLA standards, support for multiple types of interaction between simulation, large-scale QianHai, land and space tactics and drill imitation, analog signals can won 3000 x 3000 sea miles, frequency scope from 90 MHZ to $15 \mathrm{GHZ}$, to be able to access and processing dynamic communication data, including radar, and other support NH4007 data standards, such as general equipment, a variety of interface and at the time of using Amazonr cloud, as a module in the cloud computing resources to customize the execution environment, form the core automation system prototype, the system functions properly validated at the same time, establish a fixed collaboration platform, provide specific services for different users, on this basis, the data storage, access to a computer network resources, into the calculation of computing resources pool.

\subsection{Simulation training system simulation experiment}

The simulation training system is composed of the battlefield posture, which can be used in the simulation training of both real equipment and virtual equipment. The infrared guided missile simulation system, stealth tanks, destroyers and underwater targets, including flight targets, have been transferred to the monitoring server in an XML format.

Cloud computing, the first layer using single chip microprocessor storage antenna system parameter data of the PC as a server, access to the cloud layer 4, adopts client/server mode, using Socket programming realizes the server and client communication, received signal feedback to satellite antenna controller make the satellite antenna precision of star. The fifth dimensional battlefield cloud computing platform, which is juxtaposed with sea and land, can achieve precise control of satellite antennas and can be controlled remotely in the cloud. As shown in figure 1, satellite detects stealth aircraft track. 


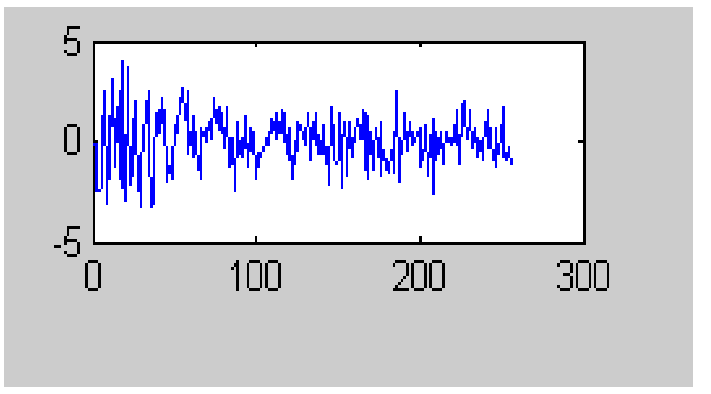

Figure 1 satellite tracking of stealth aircraft

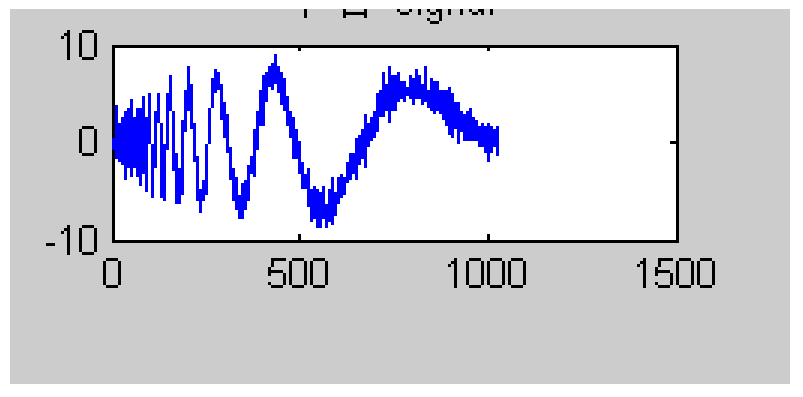

Figure 2. After cloud computing, the stealth aircraft forecasts the track

Satellite probes distinguish the different systems of radar from different platforms, which is the network topology that automatically detects infrastructure ${ }^{[4]}$ Distinguishes stealth aircraft from ships, foundations, missiles.

The simulation training system is based on the radar system to transfer the antenna parameter data through the communication platform platform to the simulation automation core system, and the system of the fourth layer prediction control and the system of position transformation are attached.

$$
\begin{aligned}
& x_{1}+x_{2}=x \\
& x_{1}+0.900008 x_{2}=x
\end{aligned}
$$

It's a typical sick system, it set: $B x=C$

The perturbation of the relative deviation of the solution to the system of the solution of the system is the disturbance of the solution $\Delta \mathrm{x}$,

$$
\frac{\|\Delta x\|}{\left\|x^{*}\right\|} \leq \frac{\left\|B^{-1}\right\| \cdot\|\Delta B\|}{2\left[1-\left\|B^{-1}\right\| \cdot\|\Delta B\|\right.}
$$

The method of surface spline interpolation algorithm is used to transform the generation of the dynamic image into a normal system, and the output data can be transmitted reliably. The simulation results are shown in figure 2

Scene graph is hierarchical, top-down tree data structure, the top is the root node of the leaf node contains form objects in the scene actual geometry information, there is a tree to free circulation, dynamic scheduling node translation, rotation and scaling, can be dynamically on the scene subtree scheduling control, combining with the ITSM for process key, in $3 \mathrm{~d}$ scene to achieve dry than letter ${ }^{[5]}$, The simulation results are very close to the actual stealth aircraft, and solve the boundary and inconsistency of the vertices in the drawing process, and the error is less than $3 \mathrm{~dB}$. Better than traditional API features

\section{Conclusion}

The generation of electromagnetic dynamics is the basis of simulation of battlefield visualization, infrastructure platform for target, first of all, to implement sea, stealth aircraft, on land and underwater signal interception of different target, through the measurement and calculation of electromagnetic scattering of land, sea and air targets ahead of the enemy found target satellite, database design and equipment carrier, automatically discover for the underlying infrastructure 
services, USB interface, realized the connection in the VS 2010 relational database, build cloud computing level, into the equipment and the calculation results of various antenna detection resulto form a tree structure, scheduling policy to cloud environment priority strategy, formed in the formation of the comprehensive evaluation on the level of land, sea and air, simulation training system simulation experiments by using scientific visualization language IDL and cloud platform environment, ITSM for key process, we complete the visualization of electromagnetic field data and the purpose of remote control freely.

\section{Acknowledgements}

Manuscript received 1 Aug, 2017. This work was supported Force NO.95275.Thanks for Fund project: 2017 national social science fund project : Research on the integration and development mechanism of China's new information network industry and military and civilian industry Name: research on the integration and development mechanism of China's new information network industry and military people.

\section{References}

[1] suri, based on the average correlation algorithm, beidou B2 signal fast capture [EB/OL] China optical journal network, 2016,

[2] wang jie, the service oriented complex electromagnetic environment electromagnetic situation visualization [D] master's thesis, nanjing university of technology, 2015

[3] zhou, the electromagnetic situation of sea battle field generated a number of key technologies to study $\{$ D $\}$, Ph.D. Dissertation, Harbin engineering university, 2013

[4] xiao hongxia, research on anti-jamming technology of satellite antenna based on multi-domain fusion [D]. Harbin engineering university, 2013:

[5] liu zengming, based on OSG's battlefield vision simulation study [D]. Xi 'an. University of electronic science and technology, 2014 